\title{
Percepción de trato digno de usuarias que acuden al servicio de interrupción legal del embarazo (ILE)
}

\section{Users' perception about worthy relationship in care of the service of legal abortion}

Mtra. Martha Lilia Bernal Becerril•

Atalia Ramírez Alba y Gabriela Ramírez••

\section{Resumen}

Las mujeres embarazadas que desean interrumpir su embarazo de forma voluntaria deben recibir un trato digno durante su atención. El Trato Digno incluye saludo amable, presentación del personal de salud, hablar a la paciente por su nombre, explicación e información de procedimientos a realizar, salvaguardar la intimidad, trato respetuoso, y la satisfacción del paciente con respecto al trato del personal que le atendió. Objetivo: Evaluar la percepción que tienen las usuarias del cumplimiento del indicador de calidad: "Trato Digno" proporcionado por personal de enfermería en el servicio de ILE. Metodología: Estudio de tipo cuantitativo, descriptivo y transversal. Muestreo por conveniencia conformado por 54 pacientes que acudieron al servicio de ILE. Resultados: La edad de las mujeres osciló entre 15 y 45 años. El 66\% de las mujeres del estudio refirieron haber esperado más de 60 minutos para recibir tratamiento; a pesar de ello 56\% reportó estar satisfecha con el tiempo de espera. El 93\% consideró que recibió información clara y concisa acerca de su tratamiento y evolución. Un porcentaje igual valora como respetuoso el trato del personal. El 89\% de las mujeres del estudio recibió cuidados de enfermería durante su atención. También 96\% dice estar satisfecha con la atención recibida. Conclusión: La atención a las pacientes que acuden al servicio de ILE corresponde a un trato digno, ya que se brinda con respeto, amabilidad, humanismo e informando acerca de los procedimientos a realizar, medidas diagnósticas y/o terapéuticas necesarias.

-Profesor Asociado B TC. UNAM ENEO.

-.Estudiantes de la carrera de Licenciatura en Enfermería y Obstetricia de la UNAM-ENEO

RECIBIDO: 22 DE ABRIL 2011

ENVIADO: 2 MAYO 2011

ACEPTADO: 31 MAYO 2011

Palabras

Clave:

Enfermería, Trato digno, Indicadores de calidad de la atención de salud, Interrupción legal del embarazo 


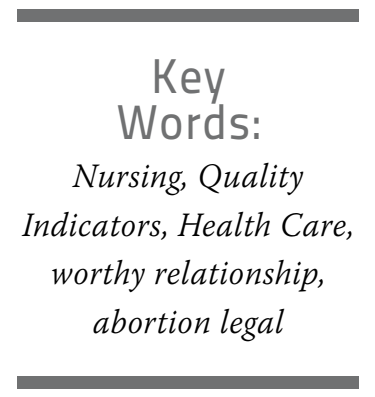

ABSTRACt

Pregnant women seeking to terminate voluntary her pregnancy on a basis should be treated with dignity in their health. The worthy relationship includes friendly greeting, presentation of professional health, and call to the patient by name, explanation and information about to make procedures, safeguard privacy, respectful, and patient satisfaction regarding the care of staff who attended her. Objective: To evaluate the users' perception of quality care indicator fulfillment with worthy relationship provided by nurses in ILE service. Methodology: quantitative, descriptive and transversal study. Convenience sample comprised of 54 patients who attended the ILE service. Results: The age of the women ranged between 15 and $45.66 \%$ of women in the study reported having waited more than 60 minutes for treatment, despite that 56\%reported being satisfied with the waiting time. 93\% felt that was clear and concise information about their treatment and evolution. The same percentage was observed respectful relationship by staff. $89 \%$ of women in the study nursing care received during your stay. $96 \%$ also say they are satisfied with the medical care. Conclusion: The care of patients attending the ILE service corresponds to a worthy relationship, as it provides with respect, kindness, humanism and report on procedures performed, diagnostic measures and / or treatment required

\section{INTRODUCCIÓN}

En México, se ha mantenido el interés por mejorar la calidad y efectividad en la entrega de los servicios de salud a la población, sana o enferma, sin importar su estatus social, raza o creencias. Éste interés está dirigido a lograr la satisfacción de los pacientes y familiares introduciendo actividades asistenciales, docentes y de investigación en cada uno de los establecimientos de salud.

Sin duda, la calidad es ya un adjetivo que debe describir la atención que brindan todos los profesionales de la salud y se ha convertido en una necesidad de mejorar la efectividad en la entrega de cuidados de salud. Ésta lucha se ve reflejada en la llamada "Cruzada Nacional por la Calidad de los Servicios de Salud" (CNCSS) que contempla varios puntos para lograr los objetivos fundamentales de promover un trato digno y adecuado y una atención médica efectiva en todas las instituciones de salud para los enfermos y sus familiares; además incluye procesos permanentes de mejora de desempeño y de transparencia, que permitirán incorporar estrategias de mejora continua.

Dentro de los servicios de salud, el trato digno que se ofrece en el marco de la CNCSS a los usuarios se refleja en: Respeto a los derechos humanos y características individuales de la persona; Información completa, veraz, y susceptible de ser entendida por el paciente o por quienes lo acompañan y Amabilidad por parte de los prestadores de servicio.

De acuerdo con la Comisión Interinstitucional de Enfermeras la calidad de los servicios de enfermería se define como: "... la atención oportuna, personalizada, humanizada, continua y eficiente que brinda el personal de enfermería de acuerdo a estándares definidos para una práctica profesional competente y responsable con el propósito de lograr la satisfacción del usuario y la del prestador del servicio".

Salvaguardar la dignidad del paciente, el respeto a su dignidad o un trato digno son las proposiciones que acompañan a la definición de unos buenos cuidados de enfermería. ${ }^{2}$ La Comisión Interinstitucional de Enfermería (CIE) ha establecido algunos Indicadores de Calidad que permitan estimar la calidad de atención de estos profesionales; dentro de los cuales se encuentra el "Trato Digno" que se refiere a la percepción que tiene el paciente o familiar del trato y de la atención proporcionada; que además es considerado como un derecho de los pacientes al recibir atención médica.

Las mujeres embarazadas que desean interrumpir su embarazo de forma voluntaria, pueden verse atormentadas por la discriminación social, prejuicios, ideologías diferentes, etc; es por tanto, responsabilidad y obligación del personal de 
salud prestador del servicio de Interrupción Legal del Embarazo (ILE), ofrecer trato digno a estas mujeres que se acercan a recibir atención médica en éste servicio.

El Trato Digno incluye el saludo amable hacia la paciente, la presentación del personal de salud, hablar a la paciente por su nombre, la explicación de actividades o procedimientos que se van a realizar, salvaguardar la intimidad de la paciente, el hacer sentir segura a la paciente, ser tratada con respeto, brindar información acerca de los cuidados posteriores al procedimiento, no solo a la paciente si no al familiar responsable, y por último, la satisfacción de la paciente con respecto al trato del personal que le atendió.

\section{Objetivo General:}

- Evaluar la percepción que tienen las usuarias del cumplimiento del indicador de calidad: "Trato Digno" proporcionado por personal de enfermería en el servicio de ILE.

\section{Objetivos Específicos:}

- Medir los indicadores de trato digno.

- Conocer las desviaciones del Indicador de Calidad de Trato Digno.

- Conocer los puntos más fuertes así como los más vulnerables para el cumplimiento del indicador de Trato Digno hacia las mujeres en el servicio de ILE.

\section{Marco Teórico}

La calidad de los servicios de enfermería se define como: “... la atención oportuna, personalizada, humanizada, continua y eficiente que brinda el personal de enfermería de acuerdo a estándares definidos para una práctica profesional competente y responsable con el propósito de lograr la satisfacción de usuario y la del prestador del servicio".

La Comisión de Arbitraje Médico (CONAMED), señala que ésta calidad se considera desde dos dimensiones: la calidad técnica que requiere de la competencia y responsabilidad del profesional para aplicar sus conocimientos y habilidades en todos los cuidados que otorga y la calidad interpersonal entendida como el Trato Digno que distingue la atención de enfermería y cuyos rasgos característicos son: comunicación, confianza y respeto.

Trato digno es el derecho de los usuarios a ser tratados como personas en toda la extensión de la palabra más que como pacientes debido a la relación asimétrica que la enfermedad o incapacidad puede establecer e involucra el derecho de libertad de movimientos, a la privacidad e intimidad por el personal médico y áreas afines del sistema de salud.

El personal de enfermería tiene la obligación, de ante cualquier paciente, ofrecer un Trato Digno, es decir, el saludo amable de la enfermera hacia la paciente, la presentación del personal de salud, hablar a la paciente por su nombre, la explicación de actividades o procedimientos que se van a realizar, salvaguardar la intimidad de la paciente, el hacer sentir segura a la paciente, el tratarlo con respeto, brindar información acerca de los cuidados posteriores al procedimiento, no solo a la paciente si no al familiar responsable, y por ultimo, la satisfacción de la paciente con respecto al trato de enfermería, son parámetros que nos ayudan a evaluar el Trato Digno.

La CNCSS admite que la atención médica sea efectiva, eficiente, ética y segura. Efectiva porque logra alcanzar los resultados esperados por el usuario de los servicios de salud; eficiente porque fomenta el uso óptimo de los recursos que se dispone; ética ya que se apega a los valores universales y segura puesto que ofrece un servicio que involucre los menores riesgos posibles.

En el otorgamiento de calidad de los servicios de la atención médica, el factor humano juega un papel fundamental al estar estrechamente relacionados con el trato personal, lo que impacta directamente el nivel de calidad con que se atiende a los usuarios. La calidad de la atención busca la manera de que produzcan el máximo de beneficios y el mínimo de riesgos para la salud del usuario.

La relación interpersonal no se mide exclusivamente por la comunicación verbal, también hace referencia al interés que se tiene por las personas, el cual se demuestra por la comodidad, privacidad y por el ambiente que se les proporciona en los diferentes lugares donde se presta la atención en salud.

El ejercicio profesional del personal que atiende cuestiones del proceso salud-enfermedad de las personas debe estar caracterizado por el respeto y cumplimiento de los valores humanos universales y los principios éticos con libertad y responsabilidad.

La evaluación de la adherencia a estos principios ha motivado el desarrollo de investigación en este campo, por ejemplo Torres-Mora y Rivas-Espinoza ${ }^{4}$ analizaron el in- 
dicador de calidad del trato digno desde la percepción del paciente, sus resultados señalan que el trato digno proporcionado por las enfermeras se consideró como bueno en $98 \%$ y regular $2 \%$ y por los enfermeros bueno $96 \%$ y regular $4 \%$, de acuerdo con la percepción del paciente el trato que proporciona el personal de enfermería es bueno sin influir el sexo del personal que atiende.

Alonso y cols. ${ }^{5}$ Por su parte se propusieron evaluar la calidad del servicio de enfermería otorgado a pacientes postquirúrgicos en un hospital de segundo nivel del Estado de México. Realizaron un estudio transversal, en 104 pacientes del servicio de ginecología (G/O) y 87 de cirugía general (CG). El trato digno excelente solo ocurrió en el $22 \%$ y $24 \%$ de los casos en G/O y CG respectivamente. En el servicio de G/O, el 77\% de los usuarios quedó satisfecho con el servicio recibido, en tanto que el 69\% lo estuvo en CG. La mayoría de ellos manifestaron haberse sentido satisfechos con el servicio que recibieron por parte del personal de enfermería.

El conocimiento del personal de enfermería sobre los derechos humanos del paciente también puede influir en el trato que se brinda y por ende en la satisfacción del usuario, conforme a esto Gutiérrez y cols. ${ }^{6}$ llevaron a cabo un estudio cuya muestra comprendió 50 enfermeras generales y 121 pacientes hospitalizados. El total del personal encuestado tiene conocimiento sobre el derecho a la salud; poco menos de la mitad (47\%) del personal conoce el derecho de trato digno. El 49\% de los pacientes refiere no conocer el nombre de la enfermera que lo atiende en los diferentes turnos y en el $51 \%$ reporta que no se respeta su intimidad. En lo que concierne a la atención de enfermería más de la mitad de los pacientes (58\%) la refiere como buena, 28\% excelente y $14 \%$ regular. El $70 \%$ de los pacientes refieren que no es respetada su decisión e intimidad respecto a su persona, ofrecimientos diagnósticos y terapéuticos. El 32\% opinó que la enfermera no se dirige a él por su nombre o apellido, al $10 \%$ no se le informa de los estudios o procedimiento que se le van a realizar, $17 \%$ no recibió un trato amable por el personal de enfermería, solo $12 \%$ considera que no es respetado su derecho en la toma de decisiones respecto a su persona, ofrecimientos diagnósticos y terapéuticos. Es decir a pesar del conocimiento respecto a los derechos de los pacientes se identificaron aspectos concretos en los que se deben realizar acciones para su mejora.

En otro estudio realizado en la Ciudad de Monterrey ${ }^{7}$ el objetivo fue identificar el cumplimiento del indicador de calidad: trato digno proporcionado por personal de enfermería con pacientes de crónicodegenerativo. Estos resultados a diferencia del estudio de Gutiérrez y cols. Mostraron un buen nivel en el trato proporcionado: el $56.1 \%$ de los encuestados refirió que "el trato digno es excelente". El 98.2\% consideró que la enfermera saluda de forma amable; se presenta ante ellos 73.7\%; se dirigen por su nombre $73.7 \%$ y $94.7 \%$ explican los procedimientos o actividades a realizar; $93 \%$ reporta que se les ofrecen condiciones que guarden su intimidad o pudor. Trata la enfermera al paciente con respeto $98.2 \%$, y prácticamente la totalidad de los encuestados (99.1\%) se siente satis- fecho con el trato.

En particular, evaluar el trato digno en mujeres que deciden una interrupción legal del embarazo, también ha despertado interés, un ejemplo es el estudio realizado por De la Fuente-Maldonado y cols. ${ }^{8}$ cuyo objetivo fue evaluar el comportamiento y actitud del personal de salud, la información proporcionada, el trato y la atención recibida; la muestra la conformaron $92 \mathrm{mu}$ jeres, asistentes a un hospital general de segundo nivel de atención de la Secretaría de Salud del Gobierno del Distrito Federal. El puntaje general obtenido por el personal médico fue de 55.6 de un máximo de 100, mientras que el personal de enfermería fue calificado con 71 puntos. Las usuarias se mostraron insatisfechas con el tiempo de espera para la consulta en 63\%; dicho tiempo de espera incumplió el indicador (menos de 30 minutos) en $82 \%$ de los casos; $74 \%$ de las encuestadas consideraron que el trato que recibieron en el hospital no fue bueno. Los parámetros con menor calificación (16\% en ambos casos) fueron la información que el médico dio a las usuarias sobre los procedimientos que se le iban a realizar y la claridad del lenguaje para explicar sobre el procedimiento y efectos secundarios.

Con base en estos antecedentes fue posible apreciar la falta de uniformidad en los resultados obtenidos y esto constituyó un aspecto de la justificación del presente estudio.

\section{Metodología}

Estudio de tipo cuantitativo, descriptivo y transversal realizado en un Hospital Materno Infantil de la Ciudad de México. El muestreo fue por conveniencia con un total de 
54. encuestas aplicadas a pacientes que acudieron al servicio de ILE en el Servicio de Salud Reproductiva durante el periodo de 8 de febrero al 29 de marzo del 2010.

Para medir el indicador de "Trato Digno", se aplicó un instrumento estructurado con veintiséis reactivos de preguntas cerradas y respuestas dicotómicas, se tomó como directriz el formato de calidad de Trato Digno ya establecido por las instituciones de salud nacional como: Secretaria de Salud, Instituto Mexicano del Seguro Social, Instituto de Seguridad y Servicios Sociales para los Trabajadores del Estado, y se agregaron reactivos que ayudaron a conocer particularidades de la población que acude al servicio de ILE.

Los criterios de selección fueron: pacientes que solicitan el servicio de ILE y que aceptaran la entrevista. Se abordó a la paciente al momento de su cita en el departamento de salud reproductiva e ILE, se le invitó a participar en la investigación donde se le proporcionó información sobre el respeto a su dignidad, se le dio a conocer que la encuesta era de tipo anónima, sin exposición de riesgos y podría suspender su participación en ella, sin ningún tipo de coacción.

Con los datos obtenidos se generó una base de datos en el programa SPSS V19, se obtuvieron resultados de estadística descriptiva, distribución de frecuencias y porcentajes.

\section{Resultados}

La muestra considerada en el estudio fue de 54 mujeres que acudieron voluntariamente al servicio de ILE y que aceptaron contestar la encuesta reconociendo su derecho de consentimiento informado.
La edad de las mujeres de la muestra osciló entre 15 y hasta 45 años de edad; $30 \%$ contaban con una edad entre 15 y 20 años y un porcentaje igual para las mujeres de entre 21 y 25 años, lo que representa que el $60 \%$ se encuentra en una edad considerada como de mayor riesgo para embarazarse por el proceso de madurez en el que se encuentra la mujer. El 59\% de esas mujeres se dedicaba al hogar, 21\% eran estudiantes y $20 \%$ comerciantes. El 41\% de las mujeres de la muestra eran solteras, el $35 \%$ se encontraba en unión libre y el 17\% eran casadas. El nivel de escolaridad con el que contaban era de un $44 \%$ para mujeres que cuentan con secundaria, 27\% con bachillerato, $16 \%$ con universidad. Para $28 \%$ de las mujeres encuestadas era su pri-

\section{Gráfica No 1}

\section{Tiempo de espera}

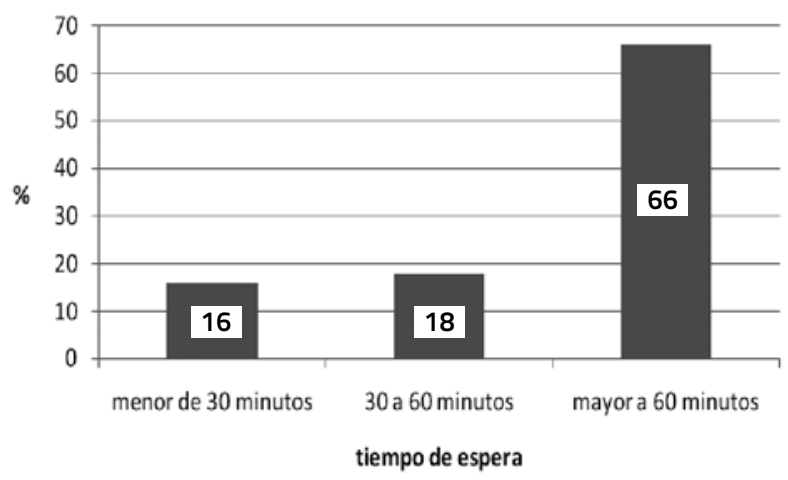

\section{Gráfica No 2 Satisfacción con el tiempo de espera.}

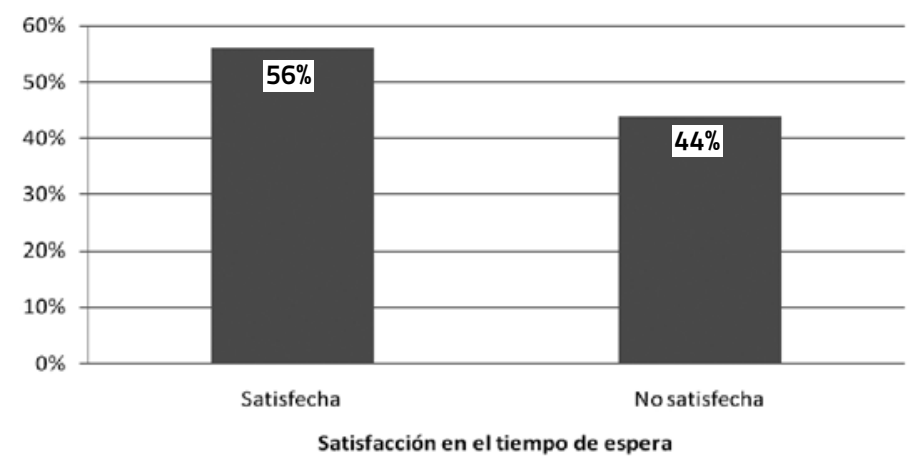


muestra asistente a ILE reportaron haber recibido información clara y concisa con respecto a su tratamiento y evolución. Así mismo el 93\% refiere haber obtenido un trato respetuoso por parte del personal que le atendió, y el 7\% dice que no recibió trato respetuoso. El 89\% de las mujeres del estudio refieren haber recibido cuidados de enfermería durante su atención y el 11\% dice no haberlos recibido. También el 96\% dice estar satisfecha con la atención recibida por parte del personal involucrado en la atención recibida en su tratamiento de ILE mientras que el $4 \%$ reporta no estar satisfecha (cuadro 1). El 89\% asegura haber recibido "buen" trato habiéndosele otorgado la información necesaria para su tratamiento y el $11 \%$ lo refiere como un trato "regular". En la evaluación del trato digno con respecto a la relación enfermera - paciente el $89 \%$ de las mujeres consideraron que esta relación fue buena, $9 \%$ que fue regular y el 2\% que fue mala (cuadro 2)

\section{DISCUSIÓN}

La evaluación del indicador de trato digno abarca diversos aspectos, uno de ellos es el tiempo de espera en la atención medica, el cual no debe de rebasar mas de 30 minutos sin importar el motivo de la consulta, condición que se ve rebasada en el presente estudio ya que hasta el $84 \%$ de las usuarias del servicio de ILE reportan tiempo de espera en la atención mayor a lo esperado para ser considerado como trato digno, condición que coincide con lo encontrado por De la Fuente-Maldo-

\section{Cuadro No. 1 Satisfacción del servicio de ILE}
Satisfaccion General
F
$\%$
Satisfecha
52
96
No satisfecha
2
4
Total
54
100

\section{Cuadro No 2 Trato Digno "Relación Enfermera-Paciente".}

Relación

Enfermera-Paciente.

Malo

Regular

Buena

Total
F

1

5

48

54
100 nado y cols. quienes señalan que en función del tiempo de espera para la consulta requerida, las usuarias se mostraron insatisfechas.

En el presente estudio un alto porcentaje reportó haber recibido información necesaria, clara y concisa con respecto a su tratamiento y evolución, situación que difiere del estudio mencionado, en él los parámetros con menor calificación fueron justamente los que dan cuenta de la información que el médico dio a las usuarias sobre los procedimientos a realizar y la claridad del lenguaje para explicar sobre el procedimiento y efectos secundarios.

Los resultados de éste estudio señalan que las pacientes que acudieron al servicio de ILE refieren, en un muy alto porcentaje, haber obtenido un trato respetuoso por parte del personal que le atendió, condición que es igual en el estudio realizado por Torres-Mora y RivasEspinosa donde se hace referencia al buen trato proporcionado por las enfermeras y enfermeros; al igual que el de Carrillo-Martínez y cols. quienes también reportan respeto en el trato y a la intimidad durante el ofrecimiento de cuidados. Cabe mencionar que el estudio realizado por Gutiérrez y cols. justamente es contrario a lo mencionado ya que hacen referencia al reporte de los pacientes que señalan que poco se respeta su intimidad y no hay un trato humanizado.

Con respecto al porcentaje de satisfacción con la atención recibida por parte del personal involucrado en la atención recibida en su tratamiento de ILE se obtuvo muy alto, y en este sentido concuerda con lo encontrado por Alonso y cols. al señalar que la mayoría de los usuarios implicados en su estudio manifesta- 
ron haberse sentido satisfechos con la atención recibida.

\section{Conclusiones}

Para la evaluación del indicador de calidad de "Trato Digno", se consideraron básicamente tres aspectos: la información que se le da a la paciente, el trato respetuoso (considerando su intimidad, y el secreto profesional) y los cuidados que reciben. En este sentido se puede señalar que durante la atención a las pacientes que acuden al servicio de Salud Reproductiva, específicamente al Servicio de ILE el trato es digno, es bueno, ya que se le trata con respeto, amabilidad, humanismo e informándole acerca de los procedimientos a realizar, medidas diagnostica y/terapéuticas necesarias de acuerdo a sus requerimientos así como de los cuidados que deben llevar en el hogar y recibiendo los cuidados pertinentes de enfermería y del personal médico para atender su condición de salud en las mejores condiciones. En general la satisfacción de las usuarias del Programa de ILE es muy alta a pesar de las complejidades propias del servicio.

Se respetan los derechos humanos como la dignidad, la intimidad, el derecho a la salud y los derechos de los pacientes incluidos en todos los sectores que prestan un servicio de salud.

El personal de salud que participa en el programa no discrimina a las mujeres que acuden a ILE, y tra$\tan$ en la medida de lo posible, que el procedimiento, ya sea por medicamento o por AMEU, no represente para la mujer una experiencia traumática.

Sin embargo, hay áreas de oportunidad de corrección específicamente en relación al tiempo de espera de las usuarias ya que se reporta como muy largo y es en lo que habrá que trabajar para reducir estos tiempos y ampliar el grado de satisfacción de las pacientes.

En ocasiones al tratar de mejorar el trato digno se enfocan estrategias de mejora en un solo ámbito, descuidado el resto de ellos, por lo que no se podría hablar realmente de una atención con trato digno en su total complejidad o integración. Así al tratar de mejorar el trato digno las estrategias se deben implementar en todos los aspectos que este aborda, dejando a un lado el hecho de que en algunos se obtenga resultados bajos.

\section{Referencias Bibliográficas}

1 Comisión Interinstitucional de Enfermería. Recomendaciones para mejorar la atención de enfermería. ISBN 970-721-222-5. México. 2003. Disponible en: http://bit.ly/ vBchBj [Consulta 10 de septiembre 2010]

2 Vecillas Sevilla MT J. La dignidad en los cuidados de salud. Ética de los Cuidados 2008 ene-jun; 1(1) España. [Internet]. Disponible en: http://bit.ly/rZYfI2 [Consulta 10 de septiembre de 2011]

3 Secretaría de Salud. Comisión Interinstitucional de Enfermería. Evaluación sistematizada de la calidad de los servicios de enfermería, 2002.

4. Torres-Mora C y Rivas-Espinosa JG. Percepción del paciente hospitalizado sobre el trato digno proporcionado por una enfermera-un enfermero. Rev Mex Enferm Cardiológica 18(3): 60-64. Disponible en: http://bit.ly/ucGS6b [Consulta agosto 2010]

5 Alonso J, González H, Pineda J, García J, Ham NZ, Corral G, Ramí- rez K, Carrillo GK. Calidad del servicio de enfermería en pacientes postquirúrgicos. Disponible en Foro institucional de investigación en salud http://bit.ly/uZ1buz [Consulta 9 de septiembre de 2010]

6 Gutiérrez S, Mederos L, Vásquez EJ, Velázquez M. Derechos del paciente hospitalizado: Responsabilidad en la práctica de enfermería. Rev Enferm IMSS 2001; 9 (1): 15-8.

7 Carrillo-Martínez M, Martínez-Coronado LE, Mendoza-López JR, Sánchez-Moreno AD, Yáñez-Torres RD, Rivera-Sáenz RV, Caballero-Escamilla R. Cumplimiento del indicador de calidad trato digno proporcionado por el personal de enfermería Monterrey, México Rev Enferm Inst Mex Seguro Soc 2009; 17 (3): 139-42.

8 De la Fuente-Maldonado M, Omaña-Martínez V, Campos-Castolo EM, Zavala-Suárez R. Evaluación de la satisfacción de mujeres que se realizan una interrupción legal del embarazo con base al indicador de Trato Digno CONAMED 2010; 15(3): 125-39. 